uninileudi identisch sei. Ich bin meinesteiles sehr viel mehr geneigt, in demselben formell entweder eine ahd. entsprechung zu got. winja, 'vour', pascuum', oder zu ıdem $s$-stamme lat. venus, ai. vánas, auslaut ahd. *wini wie sigi, mit der vielleicht in beiden fällen möglichen bedeutung 'unterhaltung, erlustigung' zu erblicken.

Demnach ist weder dieser abliegende àusdruck in das capitulare $\mathrm{zu}$ überführen, noch wie Jostes s. 313. 314 merkwürdigerweise zu glauben scheint, aus dem capitulare im wege des missverständnisses abzuleiten, sondern vielmehr anzuerkennen, dass die beiden einander äusserlich ähnlichen composita zwei nach herkunft und bedeutung verschiedene composita sind, die miteinander nicht das geringste $\mathrm{zu}$ tun haben.

CZERNOWITZ, 15. september 1910.

VON GRIENBERGER.

\title{
DIE FÄLSCHUNGEN IN DEN BEIDEN REGENS- BURGER REICHSABTEIEN OBER- UND NIEDERMÜNSTER.
}

Wie zu erwarten war, ist mein aufsatz in diesen Beiträgen 34, 143 f. 'Der Minoritenpater Bertold von Regensburg und die fälschungen in den beiden reichsabteien Ober- und Niedermünster' von einer gewissen seite der katholischen geschichtsschreibung angegriffen worden. Wie aber weiter mit wahrscheinlichkeit vorauszusehen war, hat man sich dabei durch allerhand unachtsamkeit und oberflächlichkeit schwere blössen gegeben. Da jetzt der gegner in richtige schussweite gelangt ist, soll auf diese angriffe näher eingegangen werden.

A. Väth schien so entrüstet über meine constatierung einer fälschung zu sein, dass er es zu unternehmen schien, die frommen damen ritterlich zu beschützen. Das geschah in dem Historischen jahrbuch der Görresgesellschaft 31 (1910), 39-55. Als grund für dieses so mannhafte eintreten wurde angegeben, 
ich hätte 'ohne bedenken' 'hochstehende personen', 'töchter aus den edelsten geschlechtern Bayerns zu lügnerinnen' gemacht. 'Es galt mit der feststellung der geschichtlichen wahrheit die ehre von edlen frauen aus vornehmsten familien Bayerns zu verteidigen gegenüber den schweren anschuldigungen, die grundlos gegen sie erhoben worden sind' (vgl. s. 50 und 55). Aus der schrift von Aloys Schulte, Der adel und die deutsche kirche im mittelalter; studien zur social-, rechts- und kirchengeschichte, Stuttgart 1910 = Kirchenrechtliche abhandlungen hsg. von U. Stutz, heft 63/64) erfährt man jetzt, dass Aloys Schulte der vater dieses galanten gedankens war. Väth hat in Schultes seminar die arbeit, welche meine ergebnisse 'nachprüfte', angefertigt und in erweiterter form erschien sie dann im druck (s. Schulte s. 104, anm. 1). Schulte ist natürlich zu denselben resultaten gelangt wie sein schüler (NB. zwei stimmen gegen eine!), und da es für einen lehrer immer schön ist, wenn er seinen schüler deckt, so hat er die güte, meine auf grund einer langen beschäftigung mit der frage gewonnenen ergebnisse für 'schaumschlägerei' zu erklären. Dass es sich dabei lediglich um eine deckung handelt, geht daraus hervor, dass die frage nach der fälschung mit Schultes thema gar nichts zu tun hat. Da es aber nun so sein soll, dass der lehrer auch mehr weiss als der schüler, so hat Schulte in einer anmerkung auf s. 106 noch eine sachliche bereicherung beigefügt, von der er nicht ahnte, dass er damit seine karten aufdeckte. Wenn ich also daran gehe, die oberflächliche art der untersuchung von Väth und zugleich deren echt scholastisch-jesuitische dialektik $\mathrm{zu}$ widerlegen, so wird Schulte, der sie approbiert hat, sich stets sagen müssen 'mea culpa'.

Sehen wir zunächst einmal den aufsatz von Väth rein äusserlich an. Er umfasst einen bogen zu 16 ss. + 1 s. Von diesen 17 seiten beschäftigen sich $\mathrm{s}$. 40/41 mit der geschichte der beiden abteien bis 1200. Man kann zwar nicht sagen, dass Väth hierauf die seiten $144-147$ meines aufsatzes abgeschrieben habe, aber er hat sie ausgeschrieben. Auf s. 41 bis 45 wird dann ein abdruck der auf s. 160-162 und 167/68 meines artikels veröffentlichten documente gegeben, ohne dass gesagt wird, ob sie nach einer neuen vergleichung gedruckt 
sind oder nicht. Da in diesem druck sich keine abweichungen von meinem abdruck finden, also auch die 12 von mir verbesserten lesefehler Schönbachs darin aufgenommen sind, so darf ich schliessen, dass sie entweder durch Väths nachvergleichung ihre bestätigung gefunden haben oder, was wahrscheinlicher ist, dass Väth meine texte nachdruckte. Es bleiben also von den 17 seiten des Väth'schen aufsatzes nur 11 übrig und da auf diesen entrüstungsrufe einen weiten raum einnehmen, so schrumpft der umfang dieses geschichtswerkes auf noch etwas weniger zusammen.

Auf s. 46-50 erwägt Väth, ob der commissionsbericht wolwollend sei oder nicht, und behauptet, ich hätte ihn nicht wolwollend gefunden, denn die staufergegnerische commission habe überhaupt nicht wolwollend gegen die stauferischen reichsabteien sein können. Das ist ein kampf gegen windmühlen. Ueber 'wolwollend' oder 'nicht wolwollend' bezüglich des berichtes habe ich mich gar nicht ausgesprochen. Ich habe die politischen gegensätze zwischen der antistauferischen commission und den stauferischen abteien hervorgehoben. Politische gegensätze schliessen doch noch nicht 'wolwollen' aus. Kann nicht eine politische partei, wenn sie eine andere bekämpft, trotzdem dieser wolwollend gegenüberstëhen? Ich kann ruhig bei meinen aufstellungen bleiben: 'Der bericht ist nicht gehauen und nicht gestochen', er ist 'sehr gewunden'. Und weshalb? Weil die commissionsmitglieder ihre ruhe haben wollten und nicht gerade lust hatten in so ungewissen zeiten politisch farbe zu bekennen. Deshalb brauchen die vier ehrbaren commissionsmänner noch gar keine 'feiglinge' gewesen zu sein, wie sie schwer beleidigend Väth in diesem falle bezeichnen will. Kommt es nicht auch noch heute vor, dass politisch pointierte parteimänner sich an irgend einer action ihrer partei nicht beteiligen oder nur lau, weil sie ruhe haben wollen? Man wird solche ehrbare männer doch auch heute nicht gleich 'feiglinge' nennen! ${ }^{1}$ ).

1) Wenn übrigens Väth meint, dem commissionsbericht sẹi eine eingabe der beiden stifter an den legaten vorhergegangen, so interpretiert er den bericht, wie es eben für seine zwecke passt. Vgl. s. 47. Der bericht bringt die wünsche der frauen in flehender form und hierauf beziehen sich die entsprechenden äusserungen im legatenbrief. Aus ihnen einen schluss 
Das 'papier' dieser Beiträge ist zu schade, um auf all die ergüsse Väths einzugehen. Nur ein paar charakteristische stellen sollen folgen: S. 53 heisst es: 'Da die stiftsdamen keinen anlass hatten zu fälschen, ist $B$ überhaupt nicht als fälschung in technischem sinne (!!!) des wortes aufzufassen, sondern als ein mit wissen und willen des legaten, oder wenigstens nicht gegen dessen willen (!!!) abgefasstes schriftstück' und auf s. 54: 'A ist ein amtlich beglaubigtes concept'), das an die abteien abgieng, um dort von den äbtissinnen (oder von der commission) geprüft zu werden, ob es den sachverhalt genau widergebe. Man fand daselbst, dass dies in einigen punkten nicht der fall sei und schlug verbesserungen (!) vor. Die änderungen in $B$ ergeben sich nun bei genauer prüfung wirklich als verbesserungen in dieser richtung (!). Der verfasser von A hatte die gewohnheiten auf den hl. Wolfgang zurückgeführt; das hatte der bericht nicht gesagt, er war aber gerade an dieser stelle etwas unklar gehalten, so dass wir begreifen, wie der irrtum entstehen konnte. Dieser punkt wurde also berichtigt. Auch der satz non tamen professionem absolutam facitis secundum regulam Sancti Benedicti enthält eine verbesserung, denn die schwestern waren so halb Benedictinerinnen. Die commission hatte endlich den legaten gebeten, die zarten gewissen der stiftsdamen durch eine dispens zu beruhigen; auch das wurde hinzugefügt. Daraufhin wurden

zu ziehen, ist unerlaubt. Das diu optatum im commissionsbericht ist ein viel zu allgemeiner ausdruck, den man im sinne Väths nicht pressen darf. Er wird sich auf den fall 1244 beziehen, der durch die excommunication Eberhards wider akut geworden war. Negocium aggredi heisst an einen handel herangehen, sei es um ihn über sich ergehen zu lassen, sei es um ihn selbst auszuführen. Expetita geht nicht auf ein verlangen der schwestern, sondern des legaten. Es heisst: Der legat hatte die prüfung für St. Paul nicht verlangt. In den lateinisch-mittelhochdeutschen glossaren wird expetere neben bitten und eischen mit heiszen übersetzt. Im sinne der forderung einer höhergestellten person an eine niederergestellte kommt die phrase expetere ab aliquo vor, z. b. Job•33, 34. Hrotswith 159, 11. Auch Braulio ep. 2,5. Das richtige hätten Väth und Schulte schon bei Janner 2, 420 gefunden, wenn sie geruht hätten, dieses buch nachzuschlagen.

1) Dass ich diesen punkt in erwägung gezogen hatte, konnte Väth aus s. 166 meines aufsatzes entnehmen und vermuten, dass gewichtigere gründe mich davon abgebracht hatten. Aber er und Schulte schreiben mir den fanatismus zu, den sie selbst besitzen. 
auf kosten der abteien (?) von einem deutschen schreiber zwei prachtexemplare dieser verbesserten urkunde hergestellt, dieselben noch ohne datierung in die kanzlei des legaten oder seines nachfolgers zurückgeschickt, approbiert und durch hinzufügung der datierung zum abschluss gebracht.'

Es lohnt sich gar nicht auf diese aufstellungen einzugehen, da sie, wie sich gleich zeigen wird, alle falsch sind. Nur die fälschung 'nicht in technischem sinne' möchten wir an einem modernen ähnlichen beispiel erläutern. Wenn z. b. A an B eine rechnung schickt und $B$ in gleicher form eine solche verfertigt, mit absicht aber bei dieser abschrift einen oder zwei posten weglässt, mit dieser abschrift zu A geht, diesem die auf der abschrift angegebene schuld bezahlt und sich von A die bezahlung quittieren lässt; so ist das 'überhaupt nicht als fälschung in technischem sinne des wortes aufzufassen'. -

A. Schulte hat nun s. 106, anm. 1 gemeint, ich könnte mich beruhigen, die drei klöster (Obermünster, St.Paul und Niedermünster) hätten bei der durch Nicolaus Cusanus um die mitte des fünfzehnten jahrhunderts veranlassten reform der Benedictinerklöster ihre alten freiheiten unbeeinträchtigt beibehalten. Er verweist auf die aufzeichnüngen des damals für den metropolitanbereich Salzburg als visitator bestimmten priors Johann Schlitpacher von Melk (hsg. von J. Zibermayr in den MJÖG. 30 [1909], 274). Aber was sagt diese aufzeichnung? Der bischof von Regensburg - es war Friedrich III. - hatte erklärt, er wolle wegen der angelegenheit der drei klöster sich selbst beim vatican kümmern.

Es ist eine gute alte sitte der deutschen gelehrten, dass man die bücher ganz durchliest, die über eine frage handeln, die wissenschaftlich geprüft werden soll. Für den aufsatz in diesen Beiträgen 34, $143 \mathrm{f}$. las ich seiner zeit die dreibändige Geschichte der bischöfe von Regensburg von dr. Ferdinand Janner, Regensburg 1883 f. durch, um mich über die șchịcksale der drei klöster bis $1500 \mathrm{zu}$ unterrichten. Ich hätte es nicht zu tun gebraucht. Denn in diesem trefflichen werk des katholischen gelehrten ist ein ebenso treffliches register jedem der drei bände beigegeben, in dem man unter den stichworten 'Obermünster', 'Niedermünster' und 'St. Paul' eine jede stelle, die über eines dieser klöster handelt, verzeichnet findet. Wären 
nun Väth und Schulte gründlich gewesen und hätten sie mich gewissenhaft 'nachgeprüft', so hätten sie wenigstens die register nachgeschlagen. ') Aber beide sind gar nicht auf den gedanken gekommen, obwol Schulte aus der bemerkung des Melker priors hätte sehen müssen, dass die streitfrage um 1450 keineswegs irgendwie geklärt war. Die beiden historiographen hätten dann bei Janner gelesen, dass 1479 am 1. april Sixt IV. grollend aber machtlos die gewohnheiten der drei klöster als 'durch betrug erlangt' bezeichnet hat, dass bei den acten der drei klöster zum jahre 1459 ein sehr wichtiges schriftstück liegt und dass überhaupt seit 1435 bis fast 1500 über die frage viel gestritten, viel tinte geflossen und viel papier verschrieben worden ist. Wenn ich seiner zeit auf diese dinge nicht eingegangen bin, so geschah es der kürze halber, und weil ich glaubte, dass aus der bulle von Innozenz IV. vom 11. nov. 1247 zur genüge hervorgienge, dass der päpstlichen kanzlei ein legatenbericht in der fassung $A$ vorgelegen habe, letzteres übrigens ein punkt, auf den unsere beiden historiens in ihrem ritterlichen eifer nicht weiter eingegangen sind. Ich muss also doch jetzt auf diese dinge zu sprechen kommen.

Schon unter bischof Konrad VII. (1428-37) waren in den klöstern Ober-, Nieder- und Mittelmünster zustände eingerissen, die sich in keinen stücken mit zucht und ordnung zusammenreimten. Für Obermünster musste der kirchenfürst das betreten des refectoriums, des chors und des schlafsaals durch mannspersonen verbieten. Die äbtissin wurde angehalten die schlösser und schlüssel sämmtlicher ausgänge aus dem kloster ändern zu lassen, damit weder die stiftsdamen auf der strasse herumflanieren könnten, noch herren das kloster betreten. Wie lange diese massregel genützt hat, wissen wir nicht. Als dann in die cusanische reformbewegung auch Regensburg hineingezogen wurde, antwortete bischof Friedrich III. schriftlich an den visitator, so, wie wir schon hörten. Ob etwas durch seine vorstellungen in Rom erreicht worden ist, ist unbekannt. Wir vermissen die acten darüber noch "heute, wie sie Janner vermisst hat. Nur Schultes phantasie weiss, dass in den verlorenen

1) Wie die anm. 1 auf s. 526/527 oben beweist, haben die beiden gewissenhaften nicht einmal den 2. band von Janner in die hand genommen. 
acten die bestätigung der alten freiheiten und gewohnheiten der drei klöster enthalten war. Jedenfalls war durch Friedrich III. die sache in fluss gebracht und unter dem episcopat seines nachfolgers Rupert I. geschah allerhand, worüber wir wider actenmässig unterrichtet sind. Die äbtissinnen der drei klöster hatten sich - die veranlassung kennen wir nicht mehr - an papst Calixt III. gewant mit einem exposé, in dem sie ausführten, dass nur in der legende des hl. Wolfgang die rede davon sei, dass ihre convente dem Benedictinerorden angehören. Sie schrieben, dass man in Obermünster und St. Paul mit den worten: Ego promitto obedientiam abbatisse secundum consuetudinem huius loci das gehorsamsgelübde ablege, in Niedermünster aber mit der formel: Ego promitto obedientiam abbatisse secundum formam regule sancti Benedicti abbatis pro posse secundum consuctudinem huius loci, bonan, honestam, approbatam et confirmatam. Sie erzählten weiter, dass sie ausserhalb des chores keine tracht, sondern schwarze kleider trügen und dass diejenigen, welche noch nicht das gehorsamsversprechen geleistet hätten, in bunten kleidern und gefütterten pelzen einhergiengen. Diese und einige andere dinge baten sie zu confirmieren. Der papst erinnerte sich daran, dass vor längerer zeit bischof Konrad VII. sich veranlasst gesehen habe, einzugreifen und dass bereits unter Innocenz. IV. die sache einmal akut gewesen sei. Da er aber nicht völlig sicher war, beauftragte er den Regensburger domherren und probst von Herrieden Thomas Pirkheimer mit einer prüfung der angelegenheit.

Da heisst es nun in der bulle von Pius II. vom 9. august 1459, welche uns über diese vorgänge unterrichtet:

..... et dudum tempore felicis recordationis Innocentij papae tertii (sic!) etiam predecessoris nostri per quendam tune in illis partibus apostolice sedis legatum inferioris et superioris monașteriorum predictorum abbatissis libertates et consuetudines, que eis ex translatione sancti Wolffgangi predicti prouide longis temporibus remanserant, confirmate fuerant, pro ut in ipsius legati literis plenius contineri dicebatur ...

Die cursiv gedruckten worte finden sich nur in dem sćhreiben A des legaten Philipp von Ferrara. ${ }^{1}$ ) In Rom besass man also noch in der mitte des 15. jahrhunderts

1) Der dentlichkeit halber mögen die betreffenden stellen von A und $\mathrm{BC}$ noch einmal gegenübergestellt werden: 
ein dem im Niedermünsterer archiv befindlichen legatenbrief A entsprechendes schreiben, das die gewohnheiten in Ober- und Niedermünster auf den-hl. Wolfgang zurückführte. A. Väth hatte die streichung dieser stelle in $\mathrm{BC}$ für eine verbesserung erklärt, die 'mit wissen und willen des legaten oder wenigstens nicht gegen dessen willen' vorgenommen wurde. Der legat hatte aber anders nach Rom berichtet! Es kann also gar keinem zweifel unterliegen, dass $\mathrm{BC}$ ohne wissen und willen des legaten und bestimmt gegen dessen willen fabriciert worden ist, d. h. auf gut deutsch, dass die schriftstücke BC fälschungen sind.

So wären wir schon an das ende unserer untersuchung gelangt und könnten schliessen. Aber wir haben noch allerhand schöne geschichten. zu erzählen, die unsere beiden historiographen gewiss interessieren. Die sache war noch lange nicht aus. Thomas Pirkheimer 'prüfte' die freiheiten und gewohnheiten der drei abteien und fand alles in schönster ordnung. $\mathbb{E r}$ berichtete hierüber, da inzwischen Calixt III. gestorben war, an Pius II., den früheren ratgeber kaiser Friedrichs III. Aeneas Silvius Piccolomini. Als antwort auf diesen bericht lief bei Ober-, Mittel- und Niedermünster die erwähnte bulle Pius II. vom 9. aug. 1459 ein. In ihr wurden die oben citierten gehorsamsgelübde und sonstigen gewohnheiten gutgeheissen, confirmiert und approbiert.

\section{A $(e c h t)$}

libertates et consuetudines que vobis ex translatione beati v̌ol(fgangi) prouidi et longis postea temporibus remanserunt.
BC (gefälscht)

libertates ac consuetudines que uobis" (nobis C)

longis temporibus remanserunt.

Die bulle des Pius widerlegt übrigens auch den einwand Väths auf s. 54, dass 'privilegien identischen inhalts, die für mehrere ausdrücklich genannte, rechtlich von einander getrennte unabhängige empfänger ausgestellt sind', getrennt zu adressieren seien. Sie ist an alle drei stifter zusammen adressiert und jedes dieser stifter erhielt ein solches an die drei stifter gemeinsam adressiertes exemplar. Genau so verhält “es sich mit $A$. In Obermünster ist $A$ vernichtet worden, während in Niedermünster es erhalten blieb. Wenn sich Väth über letzteren punkt wundert, so braucht man ihn bloss auf moderne verhältnisse zu verweisen. Wie häufig kommt es nicht vor, dass die mittel und vorlagen, nach denen eine fälschung hergestellt wurde, sich noch in der wohnung des verbrechers finden: z. b. fall Hofrichter. 
Man kann sich denken, was das für eine art von 'prüfung' war, die Pirkheimer vorgenommen hatte. Er hatte allen gruind, möglichst bald eine wolwollende bulle von,Pius II. zu erwirken, denn schon 1459 scheint ihm in Regensburg der boden heiss unter den füssen geworden zu seín. 'Es gieng nämlich' ich bediene mich der worte Janners 3, 519 — '1459 das gerücht, Pius II. wolle eine allgemeine versammlung der christlichen souveräne abhalten, um sie zu einer gemeinsamen action gegen die Türken zu begeistern. Pirkheimer machte nun dem rat weis, er habe den grössten einfluss auf Pius und er wolle denselben zu bestimmen versuchen, diese versammlung in Regensburg abzuhalten, jedoch habe er kein reisegeld. Der rat gab ihm richtig' nach dem Regensburger ratsbuch zweihundert gulden vnd̀ ein gut Roß, hätte gerne.40. Gulden gègolten; das alles war ganz verlorè vnd galt derselben Zeit der gulden vil gelds. Actum in der Fasten 1460. Inventus est nequam per omnia.

Ein solcher herr war àlso dieser vom papst ausersehene commissar! Es ist gar nicht ausgèschlossen, dass er sich für seinen wolwollenden bericht an die kurie auch noch eine kleine 'geldentschädigung' hat geben lassen. Aber das dicke ende kam nach. Für Pirkheimer allerdings zunächst noch nicht. Der betrügerische domherr erhielt sogar die würde einès päpstlichen protonotars. Stolz nannte er sich seitdem utriusque juris doctor et sedis apostolice prothonotarius. Im dec. 1462 hatte er sogar die hohe ehre, den apostolischen legaten Rudolph von Rüdesheim, reverendarius Sr. heiligkeit Pius II., in seinem hause zu Regensburg als gast zu haben und hier im hause Pirkheimers entschied am 3. dec. 1462 Rudolph zu gunsten Johanns II. Runsdorfer, des abtes der Benedictinerabtei St. Georg. zu Prüfening, in der zwischen diesem abt und dem bischof Rupert I. (1457-65) schwebenden streitfrage über die ecclesia parrochialis in Hennbawer 1 Regensburger diöcese. Rupert behauptete, Johannes habe hierüber eine bullam surrepticiam $\mathrm{zu}$ erlangen gewusst (Prüfening kloster fasc. 55). Damals war es wol auch, dass Rudolph die freiheiten der drei abteien auf ihre 'ordnungsmässigkeit' hin prüfte und gut befand. 1) Wie

1) Nur aus der bulle Sixts IV. vom 1. april 1479 bekannt (O. Fasc. 80). Vgl. unten. 
konnte er auch anders? Sollte er den hochwürdigen protonotar Thomas Pirkheimer blamieren?

Geholfen hat auch die bestätigung dieses apostolischen legaten nicht, denn noch vor 1468 wurde auf betreiben bischof Heinrichs IV. (1465-92) von Regensburg nochmals eine untersuchung der angelegenheit mit dem ausdrücklichen hinweis, die documente auf ihre richtigkeit hin zu prüfen, angeordnet. Man verfiel auf die geeignetste persönlichkeit: man beauftragte Johannes Runsdorfer, den abt von St. Georg, mit der 'prüfung'. 1) Sollte der hochwürdige abt den apostolischen legaten blamieren? Sollte or den apostolischen protonotarius Thomas Pirkheimer bloss stellen? Konnte es in dem $1462 \mathrm{zu}$ seinem gunsten entschiedenen handel zwischen bischof Rupert und ihm schliesslich nicht auch ihm an den kragen gehen, wenn der bischof recht bekam? Nein, abt Johann fand alles in schönster ordnung. So wusch eine hand die andere, und die ganze prüfung war weiter nichts als eine farce.

Aber bischof Heinrich war keineswegs gewillt, sich eine solche behandlung gefallen zu lassen. Er steckte sich hinter die kastenvögte der drei klöster, die herzöge Ludwig den reichen und Albrecht IV. von Bayern. Diese wanten sich wol schon ende des jahres 1467 an die kurie, indem sie ausführten, dass die drei klöster von jeher der Benedictinercongregation angehört hätten, dass sie aber auf grund von documenten, welche durch betrug erlangt seien ${ }^{2}$ ), nicht mehr nach dieser regel sich richteten. Dass sie im gegenteil ausserhalb des klosters sich aufhielten, abenteuerlich lebten, in laienkleidern einhergiengen und zwar der 'herren' wegen, dass sie an gastmählern und gesellschaften von männern und frauen teilnähmen, sich rühmten, keinem bestimmten orden anzugehören, ja sogar

1) Siehe nebenstehende anmerkung.

2) O. Fasc. 80. propter predictas et quascunque alias literas per surreptionem impetratas et processus habitos per eosdem illarum forma non seruata daretur euagandi et dissolute viųendi occasio etc. .... vt .... predictas et pro tempore existentes abbatissas et moniales eorundem monasteriorum ad obseruanciam regule sancti Benedicti teneri et jpsa monasteria dicti ordinis existere et literas ac processus huiusmodi nullius roboris vel momenti fore declararet jpsasque abbatissas et moniales ad-observandam regulam et ad exhibendum eidem episcopo litteras et processus huiusmodi per censuram ecclesiasticam et subtractionem fructuum ... compelleret. 
heiraten zu können, wenn sich eine passende partie böte. Die herzöge ersuchten den papst, die documente für ungiltig zu erklären und sie exhibieren zu lassen. Unter dem 4. februar 1468 gab Paulus II. bischof Heinrich 'den gemessenen auftrag, die adligen jungfern zur beobachtung der besagten regel durch mittel, die ihm die umstände und bescheidenheit eingäben, anzuhalten'.1) Nun begann ein intriguenspiel ohne gleichen und ein kampf auf leben und tod um diese gewohnheiten. 'Das adliche Frauenzimmer wolte sich ... mönchischen ordensregeln nicht unterwerfen, noch weniger den Heerzogen unter dem Vorwand, daß die fürstlichen Vorfahren die Münster gestiftet hätten, ein Recht über sich einräumen. Fine Königin des Frankenreichs, sagten die von Obermünster, verehren wir als unsere Stifterin und das Mittelmünster S. Paül verdankt dem hl. Wolfgang sein Daseyn. - Vom päpstlichen Stuhle war aber bereits ein Breve an den Bischof von Eichstädt ergangen, diese Sache zu verhandeln, welches Commissorium kaum ausgefertigt gewesen war, als der Kaiser die Klagstellung für eine Hintansetzung . seiner Würde erklärte, und sowol den Bischof von Eichstädt, als den yon Regensburg abmahnte, der. ihm gebührenden Entscheidung vorzugreifen.' Wo sie nur konnten, hatten sich die damen um bundesgenossen umgesehen. Den rat der stadt Regensburg hatten sie 1470 gegen die bayerischen herzöge mobil zu machen gesucht. Die herzöge widerum waren bemüht, mit hịlfe des rates die klöster unterzukriegen. Auch Pirkheimer, dem natürlich der ganze handel schwer auf die nerven gefallen war, spann seine fäden. Er hatte den fürstäbtissinnen $z u$ einer beschwerde an den apostolischen stuhl geraten, und diese waren ihm darin gefolgt. Dadurch waren bischof Heinrich die hände gebunden, die herzöge. Albrecht und Ludwig aber bis zum äussersten erbittert. Herzog Albrecht wies seine pfleger in Abach und Kehlheim an, Pirkheimer auf etwaigen reisen kein geleit zu geben, und herzog Ludwig erklärte ihn des ratstitels verlustig:

1) R. Zirngibls ... Abhandlung über die reihe und regierungsfolge der gefürsteten äbtissinnen in Obermünster 1787, s. 93.

2) C. Th. Gemeiner, Regensburgische chronik 3, 463 f. Das schreiben kaiser Friedrichs an bischof Heinrich von Regensburg ist datiert. Gråtz Mitich vor Bartlme 1470. 
Als ir etlich Zeit und Jare her unser Rate vnd Diener, auch deshalben in unserm Schutz, Scherme und Versprechnuß gewesen seit, und ir euch aber in solchem anders gehalten, dann wir getrawen ir getan soltet haben, das wir zu seinem Werth stellen, Demnach so schreiben Wir euch unsern bemeltten Rathsschutz, Scherme und Versprechnuß hiemit wieder ab in Kraft des Briefs, der geben ist an Erichtag nach Lucie 1470.

Als dies geschah, hatte sich Niedermünster bereits einverstanden erklärt, die reform sich gefallen zu lassen. Am 8. april 1470 schrieb Ludwig der reiche aus Landshut an herzog Albrecht: Niedermünster habe sich gefügt; und als am 7. märz 1472 die neugewählte äbtissin Agnes Nothaft von bischof Heinrich bestätigt wurde, hatte sie einen eid abgelegt, in dem Niedermünster ausdrücklich als ein kloster O. S. B. bezeichnet wurde. Der sieg der herzöge und des bischofs war hier vollständig und die Niedermünsterer äbtissin hatte somit ihre gewohnheiten und freiheiten als per surreptionem impetratas erklärt.

Obermünster und St. Paul blieben renitent. Die herzöge hatten am 1. juli 1470 den frater Christoph vom Franziskanerobservantenorden in sachen der reform der drei klöster nach Rom geschickt, aber schon vor dem 21. september hatten Oberund Mittelmünster den herzögen einen schutzbrief des kaisers vorgelegt, der diese vor weiterm einschreiten abhielt. Weiter wurde auf dem christlichen tage in Regensburg 1471 gearbeitet. Pirkheimer hatte es verstanden, durch einen geistesgegenwärtigen rat den gerade in den Regensburger dom einziehenden cardinallegaten Franciscus Todeschini-Piccolomini aus einer peinlichen situation $\mathrm{zu}$ befreien und hatte wol so dessen wolwollen sich gesichert. Die damen hatten die gelegenheit ergriffen, um auch bei diesem legaten ihre klagen vorzubringen. Cardinal Franz war nun zwar ein sehr liebenswürdiger herr, der auch nicht ganz unempfänglich für das weibliche geschlecht war: er schenkte den schwestern sein wolwollen, aber in der klagesache gieng nichts vorwärts. Der römische stuhl betraute noch mehrere cardinäle, deren namen wir nicht kennen, mit der angelegenheit ${ }^{1}$ ), aber noch anfang 1479 war die geschichte in der schwebe.

Unterdessen war die affäre durch ein neues vorkommnis

1) Nur aus der bulle Sixts IV. vom 1. april 1479 bekannt. 
nur noch verwickelter geworden. 1) Elisabeth von Paulstorf, äbtissin von Mittelmünster war am 16. mai 1472 gestorben. Im kloster entstanden zwei partèien, eine reformfreundliche, welche Anna Nothaft als nachfolgerin Elisabeths wählte, indesșen mit. dieser gegen die stärkere reformfeindliche partei mit ihrer candidatin Anna von Seckendorf durchfiel. Bischof Heinrịch griff ein und erklärte am 6. juni 1472 die wahl der Anna von Seckendorf für ungiltig. Bereits am 25. juni gieng eine beschw̄erde von seiten der reformfeindlichen partei nach Rom. Papst Sixtus İV. beauftragte den abt Johann von Tegernheim mit einer untersuchüng, und diesèr entsçhied für Anna Nothaft. Jahrelange streitereien giengen nun aus diesem urteil hervor: Sie hängen mit unserer frage zusammen, tragen aber nichts zur klärung bei. Die reformschraube sçheint immer schärfer bei St. Paul und Obermünster angezogen worden zu sein, bis schliesslich der kaiser selbst, vielleicht veranlasst durch den 1479 erfolgten äbtissinnenwechsel in Obermünster und der damit zusammenhängenden neubelehnung mit den regalien, in Rom vorstellig wurde ${ }^{2}$ ) und die schwestern beider klöster ihrerseits der kurie mit einer art streik drohten, wenn sie nicht unter den gewohnheiten und freiheiten leben dürften, unter denen und unter deren voraussetzungen sie ins kloster eingetreten seien.

Um einen öffentlichen scandal zu vermeiden ${ }^{3}$ ), erliess am 1. april 1479 deshalb Sixtus IV. jene grosse umfangreiche bulle, in der er den inhalt der seit der bulle von Piùs II. vom 9. aug. 1459 über die frage in der päpstlichen kanzlei vorhandenen acten recapituliert und schliesslich erklärt, dass die beiden klöster nicht angehalten werden dürften, nach der Benedictinerregel zu leben. Das umfangreiche document, das in petitsatz gut anderthalb bogen füllen würde, erzählt gedrängt und doch ausführlich den gang der dinge. Spricht von der anklage der surreptio gegen die klosterfrauen und erwähnt an keiner stelle,

1) Jånner 3, $541 \mathrm{f}$.

2) Das geht aus den worten Sixts IV. hervor: abbatissarum .... nec non .... Friderici Romanoin imperatoris .... abbatissarum et mulierum predictarum supremi defensoris et protectoris supplicacionibus inclinati ...

$\left.{ }^{3}\right)$ Nach Sixt IV. könnte die zurückführung zur Benedictinerregel nicht sine perturbatione maxima et scandalo fieri. 
dass eine endgiltige entscheidung die den frauen gemachten vorwürfe als unbegründet zurückgewiesen habe. Aber um des friedens willen u.s.w. - Am 12. mai 1479 stellte kaiser Friedrich III. den lehenbrief für die neugewählte äbtissin Sibille Paulstorfer aus, in dem er jr vnd jrs Gots haws Regalia, lehen vnd weltlichkeit.... jr gnade, freyheit, brieue, priuilegia, hanntfesten, recht, gerechtigkeit, altherkomen vnd gut gewohnheit in allen vnd iglichen jrenn Wortten, Puncten, Articuln, Clausulen, jnnhaltungen, meynungen vnd begreiffungen, als ob die alle vnd yede besonnder von wort zu wort in disem vnnsern leiserlichen brieue geschriben stunden, erneute, confirmierte und bestätigte und denen, die seinem befehl zuwiderhandeln würden, eine Pön von hundert Marck lotigs goldes androhte. Zugleich gebot er der neuen äbtissin bis spätestens zum 24. august 1479 dem hochgeborenenn Albrechten Pfaltzgrauen bey Reyne vnd Hertzogen in Beyren, vnserm lieben Oheim vnd fursten, gewondlich glubde vnd Eyde tun. Papt Sixtus bestimmte dann noch am 7. juli desselben jahres, dass bei der benediction der äbtissinnen der beiden klöster Obermünster und Mittelmünster, die unter speciellem schutze des kaisers stünden, nichts an die Benedictinerregel erinnern dürfe, damit kein neuer streit zwischen den klöstern und dem bischof entstehe. Die benediction habe vielmehr nach römischem ritus zu geschehen. St. Paul und Obermünster hatten also gesiegt. Nicht weil sie etwa recht bekommen hätten, sondern weil der gesetzlich höchste beschützer ein machtwort sprach, und Sixt IV. so klug war, wegen ein paar klostergewohnheiten sich nicht mit Friedrich III. zu überwerfen. Es gab wichtigeres und wertvolleres, worüber man mit diesem kaiser feilschen konnte!

Unsere beiden historiographen werden sehen, dass sie sich eigentlich ein bischen lächerlich mit ihrer Don Quixoterie gemacht haben. Ich befinde mich in einer äusserst vornehmen gesellschaft. Ein bischof von Regensburg hat die in frage stehenden gewohnheiten als widerrechtlich bekämpft und zwei bayerische herzöge haben die briefe, auf denen sie fussen, als durch betrug erlangt erklärt. Zwei bayerische herzöge haben dasselbe was ich getan, haben 'töchter aus den edelsten geschlechtern Bayerns' zu betrügerinnen gestempelt und Se. Heiligkeit Sixt IV. hat weder ja noch nein dazu gesagt, sondern 
bureaukratisch den vorwurf in einer wolwollenden bulle objectiv registriert. Auf seiten unserer beiden geschichtsforscher steht ein vom rat der stadt Regensburg und zwei bayerischen herzögen als lump erkannter domherr und zwei mit ihm mehr oder weniger zusammenhängende männer. Ja die beiden herzöge haben sogar noch weiter dasselbe getan wie ich; denn dadurch, dass sie die gewohnheiten der klöster als auf betrug fussend erklärten, declarierten sie auch den commissionsbericht für betrügerisch, sei es nun dass sie meinten, die commission habe betrogen oder die commission sei betrogen worden. Ich habe den bericht als nicht gehauen und nicht gestochen bezeichnet und bleibe dabei. Denn ich kann mich nicht entschliessen zu glauben, dass Bertold von Regensburg wissentlich die unwahrheit gesagt habe. Ich bin noch immer davon überzeugt, dass die commission an der nase herumgeführt wurde, dass die beiden convente gelogen haben, dass sie vielleicht sogar meineidig sind.

Auch die politischen fragen, die sich im laufe der jahrhunderte an die streitfrage geknüpft haben, sind im wesentlichen dieselben geblieben. Die kurie hat kein einziges mal bis zur bulle Sixts IV. vom 1. april 1479 ihren standpunkt geändert. Sie hat immer die ansicht vertreten, dass die zustände in den drei klöstern auf den hl. Wolfgang zurückgiengen und der bischof von Regensburg auf grund dieser tatsache ein approbationsrecht habe. Kaiser Friedrich III. stellte sich auf denselben standpunkt, den seiner zeit der commission gegenüber die beiden reichsabteien. Ober- und Niedermünster eingenommen haben werden, als sie diese anlogen. Wenn 1470 Niedermünster die Benedictinerregel annahm, so geschah dies entweder freiwillig oder weil man das hier noch befindliche echte legatenschreiben für soweit beweiskräftig hielt, dass der bischof von Regensburg in bezug auf das leben der Niedermünsterer nonnen ein aufsichts- und approbationsrecht besitze. Gewiss unrecht war es aber, dass St. Paul der Benedictinerregel entzogen wurde. Dieser punkt allein zeigt schon, dass bei der gemeinschaftlichen regelung der streitigkeiten durch kaiser Friedrich III. und Sixt IV. nicht kirchenrechtliche fragen ausschlaggebend waren, sondern das practische verlangen, nach dem langen ergebnislosen processieren anständige, 
friedliche und geordnete zustände in den klöstern einzuführen.

Dass unseren beiden historiographen meine auffassung des ganzen falles 1246 als einer finanzoperation Heinrich Raspes absolut nicht passt, lässt sich bei ihrem standpunkt durchaus begreifen. Dass der fall etwas ganz extraordinäres war, bezeugen die langen streitigkeiten darüber. Gerade von berufener seite wurde mir seiner zeit geschrieben, ich hätte mit recht den engen zusammenhang zwischen dem legaten und Heinrich Raspe betont. Der legat entschied zu gunsten des Regensburger bischofs, er berief sich auf die person des hl. Wolfgang und damit entschied er auch im sinne seiner partei. Noch im 15. jahrhundert hatte man seinen bericht, der die consuetudines auf eine duldung durch den hl. Wolfgang zurückführte, in der päpstlichen kanzlei. Zwischen 1248 und 1310 ist dieser bericht verfälscht worden. Wir sehen uns in keiner weise veranlasst, irgend eines unserer worte zurückzunehmen.

Da unsere beiden historiographen aber die geschichte vom standpunkte der moraltheologie aus betrachten, so wollen auch wir mit ein paar worten darauf eingehen. Wir halten diese fälschungen für nicht so schlimm, wie unsere beiden geschichtskundigen. Die kirche hatte im mittelalter ganz andere fälschungen auf dem gewissen. Die fürstäbtissinnen sind seiner zeit ihrem könig und dem ihm geleisteten eid treu geblieben. Das stellen wir moralisch höher, als wenn sie sich dem legaten und seiner creatur, Heinrich Raspe, gefügt hätten und auf diese weise meineidig geworden wären. Unsere beiden historiens werden darüber allerdings anders denken. Aber wir möchten ihnen raten: bevor sie die constatierung einer fälschung 'widerlegen', sich etwas gründlicher in der literatur umzusehen. Das ist die gediegendste grundlage für jede wissenschaftliche arbeit.

$$
\text { MÜNCHEN, im october. FRIEDRICH WILHELM. }
$$

